Sādhanā Vol. 37, Part 6, December 2012, pp. 739-745. (C) Indian Academy of Sciences

\title{
Automated image enhancement using power law transformations
}

\author{
S P VIMAL ${ }^{1}$ and P K THIRUVIKRAMAN ${ }^{2, *}$ \\ ${ }^{1}$ Birla Institute of Technology \& Science (BITS), Pilani 333 031, India \\ ${ }^{2}$ Birla Institute of Technology \& Science (BITS), Pilani, Hyderabad Campus, \\ Hyderabad 500 078, India \\ e-mail: thiru@bits-hyderabad.ac.in
}

MS received 10 February 2012; revised 17 June 2012; accepted 18 September 2012

\begin{abstract}
We propose a scheme for automating power law transformations which are used for image enhancement. The scheme we propose does not require the user to choose the exponent in the power law transformation. This method works well for images having poor contrast, especially to those images in which the peaks corresponding to the background and the foreground are not widely separated.
\end{abstract}

Keywords. Image enhancement; power law transformations; contrast stretching.

\section{Introduction}

Image enhancement (Gonzalez \& Woods 2005) is one of the basic problems in Image processing. It also forms the first stage in the pre-processing of images which have to be subjected to image recognition algorithms (Sonka et al 1999). Even though a variety of methods exist for Image enhancement, including power law transformations, histogram specification, etc, many of them require user input. Methods which do not require user input, for example, histogram equalization may sometimes fail for certain class of images (Gonzalez \& Woods 2005).

Automatically enhancing contrast of an image has been a challenging task since the digital image can represent variety of scene types. Trifonov et al (2001) performed automatic contrast enhancement by automatically determining the measure of central tendency of the brightness histogram of an image and shifting and transforming the histogram appropriately. Starck et al (2003) proposed a curvelet-based contrast enhancement algorithm which exploited curvelet properties for contrast stretching for efficient enhancement of contrast of an image. Their method works well for an image in the presence of noise. Stark (2000) proposed cumulative functions to be used in conjunction with histogram equalization for achieving contrast enhancements. This cumulative function is a parameter whose tuning gives varying degree of contrast enhancement. We propose a simple method for automatically enhancing the contrast of the given image. We propose an efficient method to choose the parameters of power law transformation automatically.

*For correspondence 


\section{Power law transformations}

Image enhancement can be carried out in the spatial or the Fourier domains and one of the important parameters to be looked at in this context is contrast enhancement. In the spatial domain, the methods used may be further classified into: gray level transformations, histogram processing, etc. As mentioned above, histogram equalization suffers from the fact that it may sometimes decrease the contrast. Histogram specification or matching can be tailor-made to suit the images but requires lot of user input. Similarly, power law transformations or piece-wise linear transformation functions also require lot of user input. In the former case one has to choose the exponent appearing in the transformation function, while in the latter case one has to choose the slopes and ranges of the straight lines which form the transformation function.

The power-law transformation is usually defined as

$$
s=c r^{\gamma},
$$

where $\mathrm{s}$ and $\mathrm{r}$ are the gray levels of the pixels in the output and the input images, respectively and $\mathrm{c}$ is a constant. These power law transformation functions are shown graphically in the diagram (figure 1).

Figure 1 shows the plot of power law transformation with the input gray level $\mathrm{r}$ along the $\mathrm{x}$ axis and the output gray level s on the y axis for various values of $\gamma$.

We will now consider that these transformations are applied on a low contrast image. We assume that the background and foreground peaks have almost merged together in such a low contrast image. Let $r_{\max }$ be the dominant peak in the histogram. If $r_{\max }$ has a large value (in the range 150-255), then it is seen from the above graph that contrast stretching occurs by choosing $\gamma>1$ whereas for dark images ( $\mathrm{r}_{\max }$ lies in the range $\left.0-100\right)$, we see that choosing $\gamma<1$ leads to contrast stretching. We can of course, automate this procedure for choosing the appropriate exponent, by finding out the peak value in the histogram. However, we can further generalize this procedure so that the most appropriate value of the exponent is chosen.

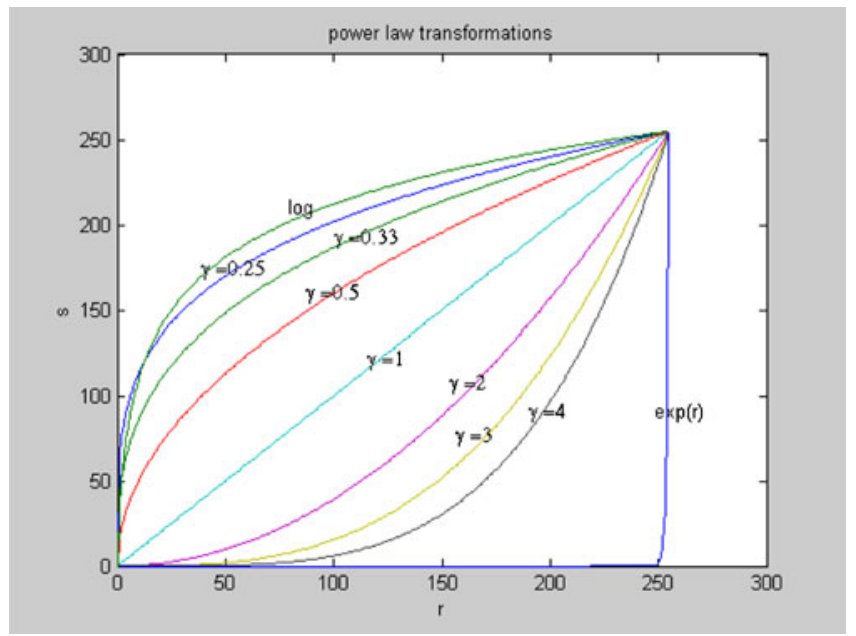

Figure 1. Plot of power law transformation for various $\gamma$ with input gray level along $\mathrm{X}$ axis and output gray level along Y axis. 


\section{Automated image enhancement}

We realize from the above discussion that maximum contrast stretching occurs by choosing the value of $\gamma$ for which the transformation function has the maximum slope at $r=r_{\max }$.

That is, if $\mathrm{m}$ is the slope of the transformation function, then we should find the value of $\gamma$, which results in the maximum value of $\mathrm{m}$ at $r=r_{\max }$.

Since we would usually like both $s$ and $r$ to run from 0 to $L-1$, we should have,

$$
c=(L-1)^{1-\gamma}
$$

Now,

$$
m=\frac{d s}{d r}=\gamma c r^{\gamma-1}
$$

We would like to maximize $m$ by varying $\gamma$. Therefore, the required condition is

$$
\begin{gathered}
\left.\frac{d m}{d \gamma}\right|_{r=r_{\max }}=0 \\
\left.\frac{d m}{d \gamma}\right|_{r=r_{\max }}=\left(\frac{r_{\max }}{L-1}\right)^{1-\gamma}+\gamma\left(\frac{r_{\max }}{L-1}\right)^{1-\gamma}=0 .
\end{gathered}
$$

Therefore

$$
\gamma=-\frac{1}{\ln \left(\frac{r_{\max }}{L-1}\right)} .
$$

Hence, given the value of $r_{\max }$, which corresponds to the peak in the histogram, we can determine the corresponding value of the exponent which would maximize the extent of the contrast stretching.

\section{Testing of the algorithm}

We selected an image which had a single dominant peak in the histogram and subjected it to the enhancement algorithm described above. The value of $r_{\max }$, as determined by the program (written in MATLAB) was found to be 164. The corresponding value of gamma is found to be 2.2655. The original and the enhanced images are reproduced below showing clearly that the algorithm works for images which have a single dominant peak.

In order to quantitatively verify the improvement in contrast, we calculated the standard deviations of the input and the output images and found them to be 48.59 and 52.23, respectively. This confirms the improvement in contrast, if standard deviation of the gray level of the pixels is taken as a measure of contrast.

Some precautions have to be taken in using the algorithm in order to avoid some computational problems. In case the value of $r_{\max }=0$, then the corresponding value of $\gamma$ is 0 . Using this value would lead to erroneous results since, all values of $r$ (input gray level) would be mapped to the same value of $s$ (output gray level). In this case, the program checks whether the peak occurs at 0 and if so replaces, the power law transformation by a logarithmic transformation.

A similar problem occurs if the peak is at L-1 (i.e., 255 in the case of a 8-bit grayscale image). In this case, the power law transformation is to be replaced by an exponential transformation. In fact, in our algorithm, we have replaced the power law by an exponential transformation, 
whenever $r_{\max }>250$, as otherwise the gamma value which is computed by the algorithm is larger than the maximum number which can be handled by the computer.

In the recent past, efforts have been made to automate image enhancement using neural networks (Jun et al 2008). Our algorithm has the advantage that it is very simple to implement and does not have any arbitrary parameters which have to be fixed by the user.

\section{Results for images with multiple peaks in the histogram}

The above algorithm assumes that the input image is a low contrast image with a single peak. However, in practice, most images have at least two peaks, one corresponding to the foreground and one corresponding to the background. If we use the same approach for images with two peaks, the results still show marginal improvement, though the improvement is not as impressive as in the case of figure 2, as the proposed approach works around the most dominant peak. We applied our algorithm on images with two peaks. One such image is shown in figure 3 and another in figure 5. The histogram of the input and output images are shown in figure 4 and figure 6 , respectively. We can observe that the standard deviation actually decreases marginally in both the cases on application of this algorithm. This is mainly because, in both the cases, $r_{\text {max }}$ happens to be near 0 . Hence the gamma value which is computed by the algorithm will be less than 1. As a result, the dominant peak will be shifted towards higher gray levels, thereby leading to a decrease in the standard deviation. It is to be noted that even standard algorithms

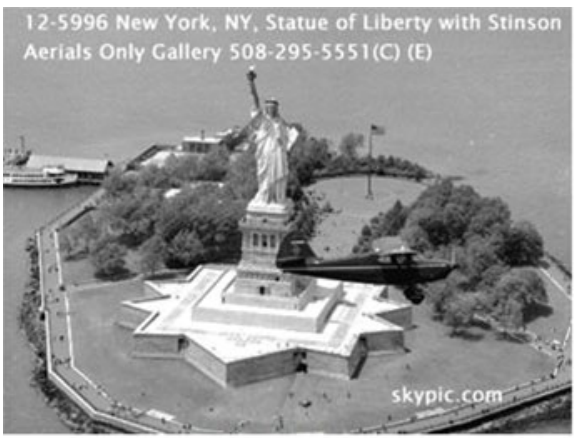

(a) Original image with a slightly washed-out apperance

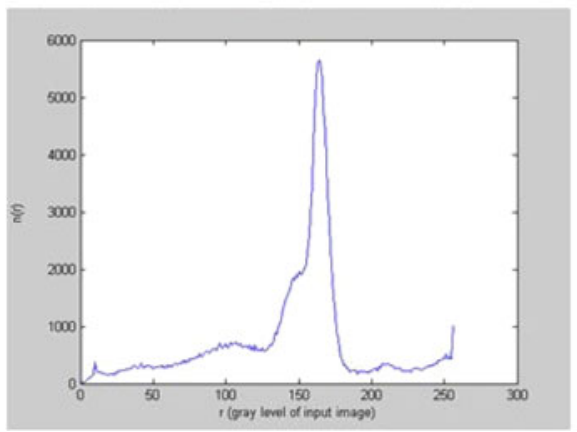

(c) Histogram of original image

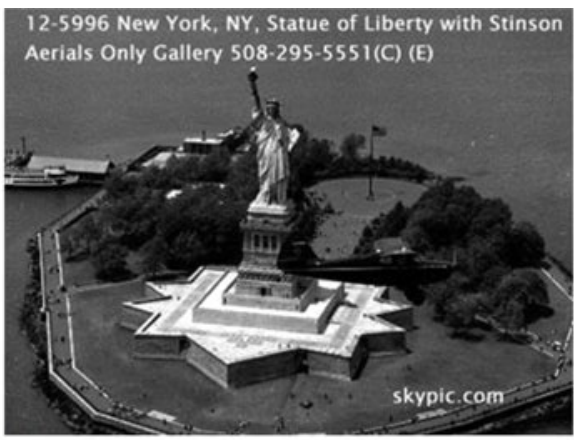

(b) Enhanced image using the automated power law transformation

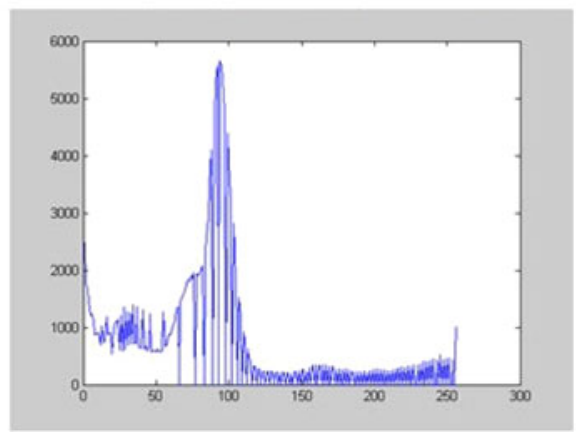

(d) Histogram of enhanced image

Figure 2. Input and output images with the respective histograms. 

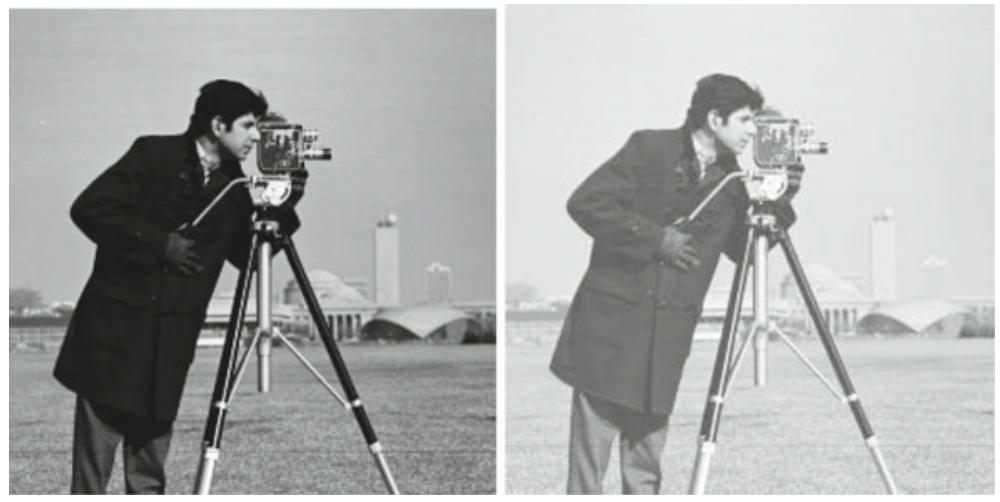

Figure 3. A test image with multiple peaks in the histogram. The input image is on the left, while the output image is on the right.
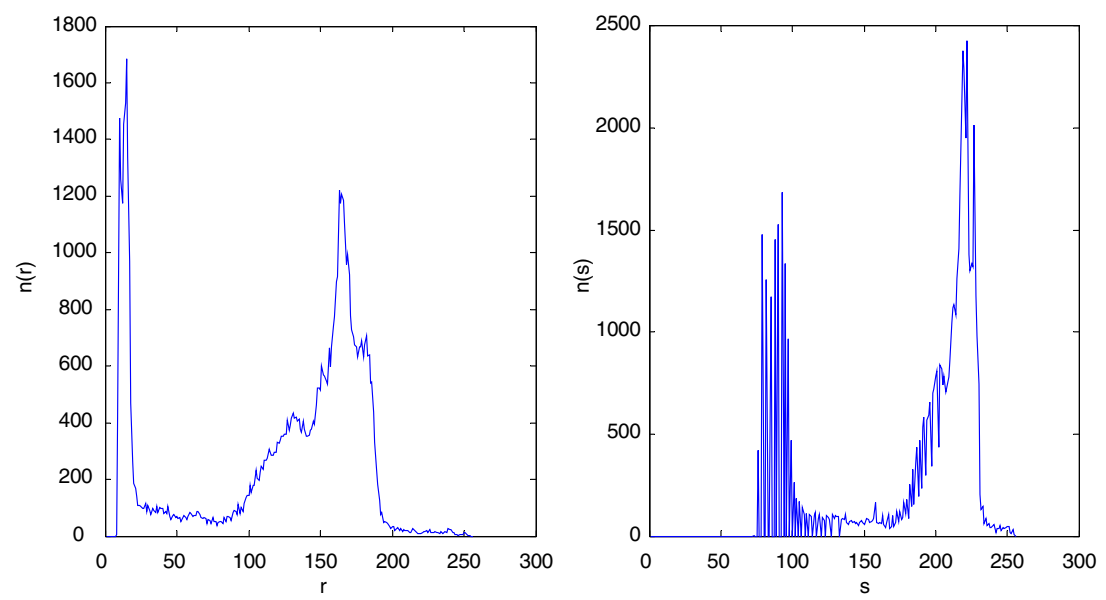

Figure 4. The histograms of the input (left) and the output (right) images shown in figure 3 . The corresponding standard deviations are 62.34 and 51.36 .
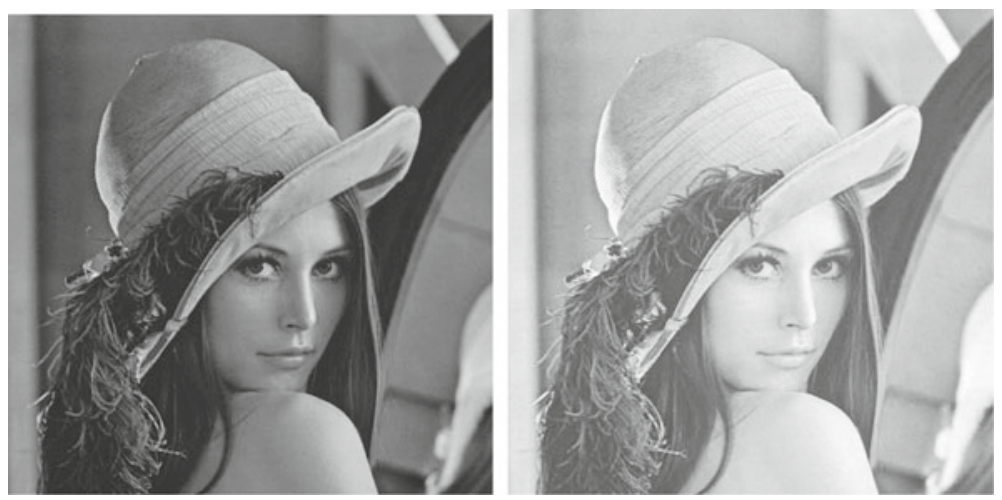

Figure 5. Test image which has multiple peaks in the histogram. The input image is on the left, while the output image is on the right. 

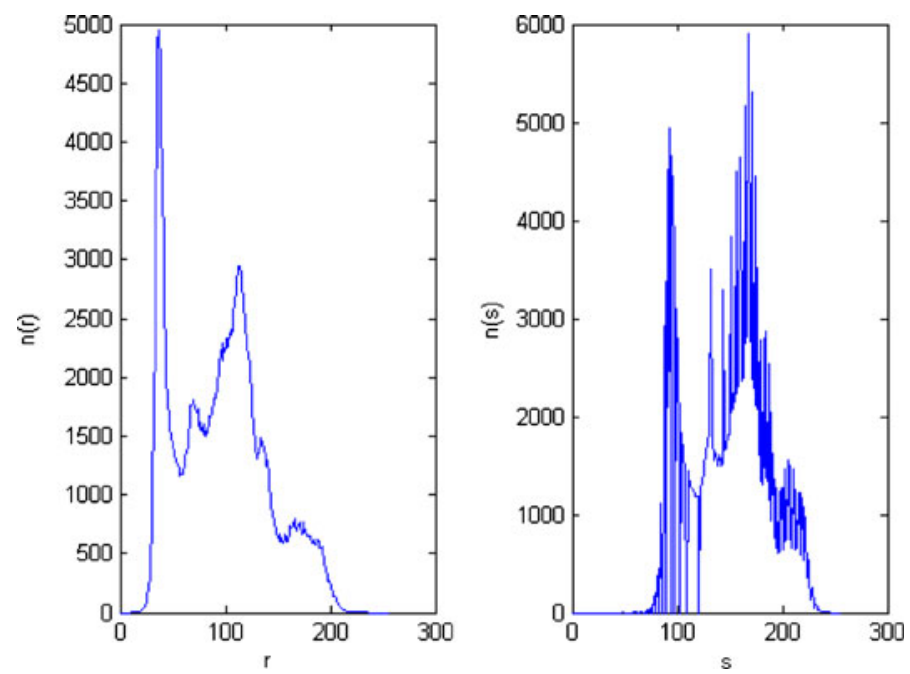

Figure 6. Histograms for the images shown in figure 5. The histogram of the input image is on the left and the histogram of the output image is on the right. The standard deviations of the input and output images are 43.89 and 37.61 , respectively.

for image enhancement like histogram equalization, fail for certain class of images (Gonzalez \& Woods 2005). Our algorithm works well in those where it is most needed, i.e., in cases, where the images have poor contrast. In cases, where the images already have good contrast (figure 3 and figure 5), the algorithm does not enhance the contrast.

We present one final example of an image with multiple peaks in the histogram. Figure 7 shows the input and output images and figure 8 shows the corresponding histograms. In this case, there is a slight improvement in contrast. As mentioned earlier, the algorithm works in this case, as the input image is of low contrast and the foreground and background peaks in the histogram are quite close to each other (unlike in the case of figures 3 and 5). In case of images similar to figure 3 or figure 5, the user may require to enhance only a particular range of gray levels (either the foreground or the background). This of course, calls for intervention by the user.
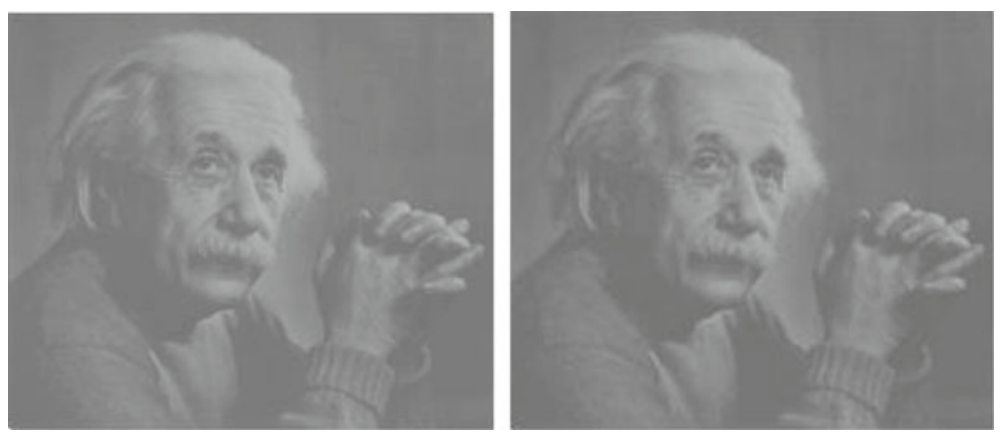

Figure 7. Example of an image with multiple peaks. The input image is on the left, while the output image is on the right. 

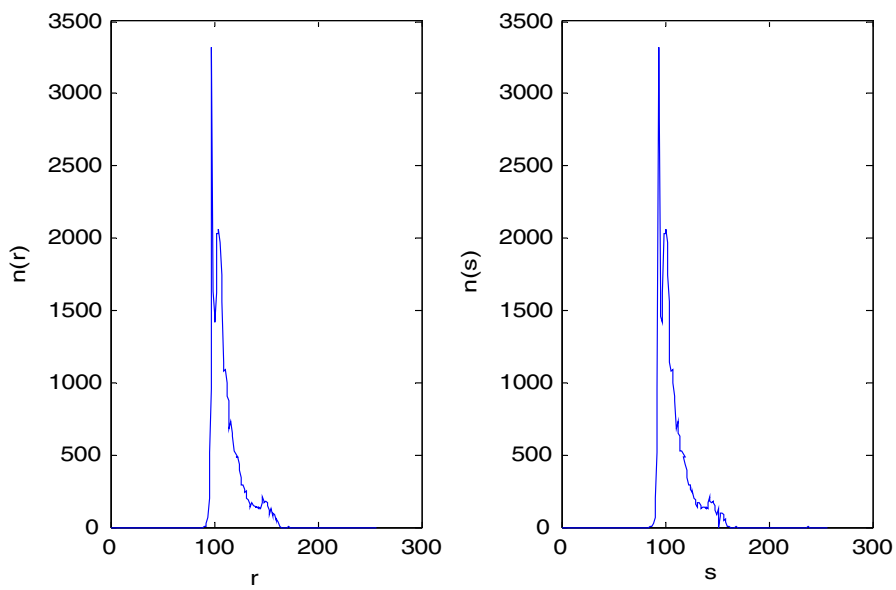

Figure 8. The input (left) and output (right) histograms for the images in figure 7. The standard deviations of the input and output images are 14.14 and 14.18, respectively.

\section{Conclusion}

We have presented a simple and efficient algorithm for automatic image enhancement and tested it on some images. The method works well for cases in which the foreground and background peaks are not well-separated, i.e., the input image is of a low contrast. In case of images having high contrast, especially those which have a wide separation between the foreground and the background peaks, the method is not so effective. In the case of such images, user intervention is required to enhance or stretch a particular range of gray levels.

\section{References}

Gonzalez R C and Woods R E 2005 Digital image processing, II Edition, New Delhi, India: Pearson Education

Jun J, Jun C and Xinglin C 2008 CISP, vol. 3, Congress on Image and Signal Processing, Volume 3, 289-293

Sonka M, Hlavac and Boyle 1999 Image processing, analysis, and machine vision, II Edition, Singapore: Brooks/Cole

Stark J A 2000 Adaptive image contrast enhancement using generalizations of histogram equalization. IEEE Trans. Image Process. 9: 889-896

Starck J, Murtagh F, Candes E and Donoho D 2003 Gray and colour image contrast enhancement by the curvelet transform. IEEE Trans. Image Process. 12(6): 706-717

Trifonov M I, Sharonova O V and Zaklika K A 2001 Automatic contrast enhancement. US Patent number: 6826310, Filing date: 6 Jul 2001, Issue date: 30 Nov 2004. Application number: 09/900,744 